\title{
Phosphorylation of ERK/MAP kinase is required for long-term potentiation in anatomically restricted regions of the lateral amygdala in vivo
}

\author{
Glenn E. Schafe, ${ }^{1,6}$ Michael W. Swank,, ${ }^{2}$ Sarina M. Rodrigues, ${ }^{3}$ Jacek Dębiec, ${ }^{3,4}$ \\ and Valérie Doyère ${ }^{3,5}$ \\ ${ }^{1}$ Department of Psychology and Interdepartmental Neuroscience Program, Yale University, New Haven, Connecticut 06520, USA; \\ 2 Division of Neuroscience, Baylor College of Medicine, Houston, Texas 77030, USA; ${ }^{3}$ W.M. Keck Foundation Laboratory \\ of Neurobiology, Center for Neural Science, New York University, New York, New York 10003, USA; ${ }^{4}$ Department of Psychiatry, \\ New York University School of Medicine, New York, New York 10016, USA; ${ }^{5}$ AMC, CNRS-UMR8620, Université \\ Paris-Sud, 91405 Orsay, France
}

\begin{abstract}
We have previously shown that the extracellular signal-regulated kinase/mitogen-activated protein kinase (ERK/ MAPK) is transiently activated in anatomically restricted regions of the lateral amygdala (LA) following Pavlovian fear conditioning and that blockade of ERK/MAPK activation in the LA impairs both fear memory consolidation and long-term potentiation (LTP) in the amygdala, in vitro. The present experiments evaluated the role of the ERK/MAPK signaling cascade in LTP at thalamo-LA input synapses, in vivo. We first show that ERK/MAPK is transiently activated/phosphorylated in the LA at $5 \mathrm{~min}$, but not 15 or $60 \mathrm{~min}$, after high-frequency, but not low-frequency, stimulation of the auditory thalamus. ERK activation induced by LTP-inducing stimulation was anatomically restricted to the same regions of the LA previously shown to exhibit ERK regulation following fear conditioning. We next show that intra-LA infusion of UO126, an inhibitor of ERK/MAPK activation, impairs LTP at thalamo-LA input synapses. Collectively, results demonstrate that ERK/MAPK activation is necessary for synaptic plasticity in anatomically defined regions of the LA, in vivo.
\end{abstract}

Several lines of evidence have suggested that long-term potentiation (LTP) in the lateral nucleus of the amygdala (LA) is a potential mechanism by which fear memories are formed and stored in the amygdala (Maren 1999; Blair et al. 2001; Schafe et al. 2001). This has been best demonstrated in the auditory fearconditioning paradigm, in which a tone is paired with footshock. LTP has been demonstrated in each of the major sensory input pathways that are known to be important for auditory fear conditioning (Chapman et al. 1990; Clugnet and LeDoux 1990; Maren and Fanselow 1995; Huang and Kandel 1998; Weisskopf and LeDoux 1999), LTP induction at auditory thalamic inputs to the LA has been shown to augment the processing of natural auditory information in the LA (Rogan and LeDoux 1995), and auditory fear conditioning induces neurophysiological changes in the LA that are similar to artificial LTP induction (McKernan and Shinnick-Gallagher 1997; Rogan et al. 1997; Schafe et al. 2005b). Further, auditory fear conditioning and LTP have been shown to be subserved by similar stimulus contingencies (Bauer et al. 2001) and pharmacological mechanisms (Huang et al. 2000; Schafe et al. 2001). However, despite the extensive interest in the link between LTP in the amygdala and fear conditioning, we still know relatively little about the biochemical mechanisms that underlie LTP in the amygdala and how, if at all, these mechanisms relate to fear memory formation.

Most studies that have examined the link between LTP and fear memory formation have done so using in vitro slice methods

\footnotetext{
${ }^{6}$ Corresponding author.
}

E-mail glenn.schafe@yale.edu; fax (203) 432-7172.

Article is online at http://www.learnmem.org/cgi/doi/10.1101//m.746808.
(Chapman et al. 1990; Huang and Kandel 1998; Weisskopf and LeDoux 1999; Huang et al. 2000; Schafe et al. 2000, 2005a; Bauer et al. 2001, 2002). The slice preparation is convenient for pharmacological and whole-cell analysis studies and allows for rapid examination of the role of different molecular signaling cascades in amygdala LTP. The slice is less optimal, however, for immunohistochemical, biochemical, and gene expression analysis experiments, all of which will be critical for examining the molecular mechanisms that underlie LTP and what these mechanisms share in common with memory formation in the intact animal. Further, the actual input pathways stimulated in the amygdala slice preparation are not well defined, making it difficult to conclude with certainty that synaptic plasticity is being measured in a behaviorally relevant input pathway. Finally, the time course of LTP decay in slice experiments following disruption of intracellular cascades that are critical to synaptic plasticity is much faster than that observed for memory itself under the influence of the same treatments, complicating the interpretation that amygdala LTP might be causally related to fear memory formation. Accordingly, it has become clear that studies employing in vivo amygdala LTP will be necessary to more thoroughly bridge the gap between behavioral studies of memory formation and those of synaptic plasticity.

We have recently shown that the extracellular signalregulated kinase/mitogen-activated protein kinase (ERK/MAPK) is transiently activated in anatomically defined regions of the lateral amygdala (LA) following Pavlovian fear conditioning and that blockade of ERK/MAPK activation in the LA impairs both fear memory consolidation and amygdala LTP, in vitro (Schafe et al. 2000). Here, we examine the role of ERK/MAPK in LTP in the LA, in vivo. 


\section{Results}

High-frequency stimulation of thalamic inputs transiently induces ERK/MAPK phosphorylation in anatomically restricted regions of the LA

In the first experiment, we used Western blotting and immunohistochemical methods to examine the regulation of ERK/MAPK following LTP-inducing stimulation of thalamic inputs to the LA. We focused on thalamic inputs because this pathway has been the most extensively characterized at the pharmacological level and has recently been shown to exhibit the longevity properties in LTP that are desirable as a mechanism of long-term memory formation (Doyère et al. 2003). In these initial experiments, rats received high-frequency stimulation (HFS) of thalamic inputs to the LA as described below (see Materials and Methods) and were sacrificed by decapitation or perfusion immediately (within 5 $\mathrm{min}$ ), $15 \mathrm{~min}$, or $60 \mathrm{~min}$ later. The 5 - and 60-min time points have previously been shown to be the optimal times at which ERK/MAPK is regulated in the LA following fear conditioning (Schafe et al. 2000; Paul et al. 2007).

The results of the Western blotting experiments are presented in Figure 1, B and C. High-frequency stimulation induced a robust activation of both ERK1 (phospho-ERK1; Fig. 1B, top)

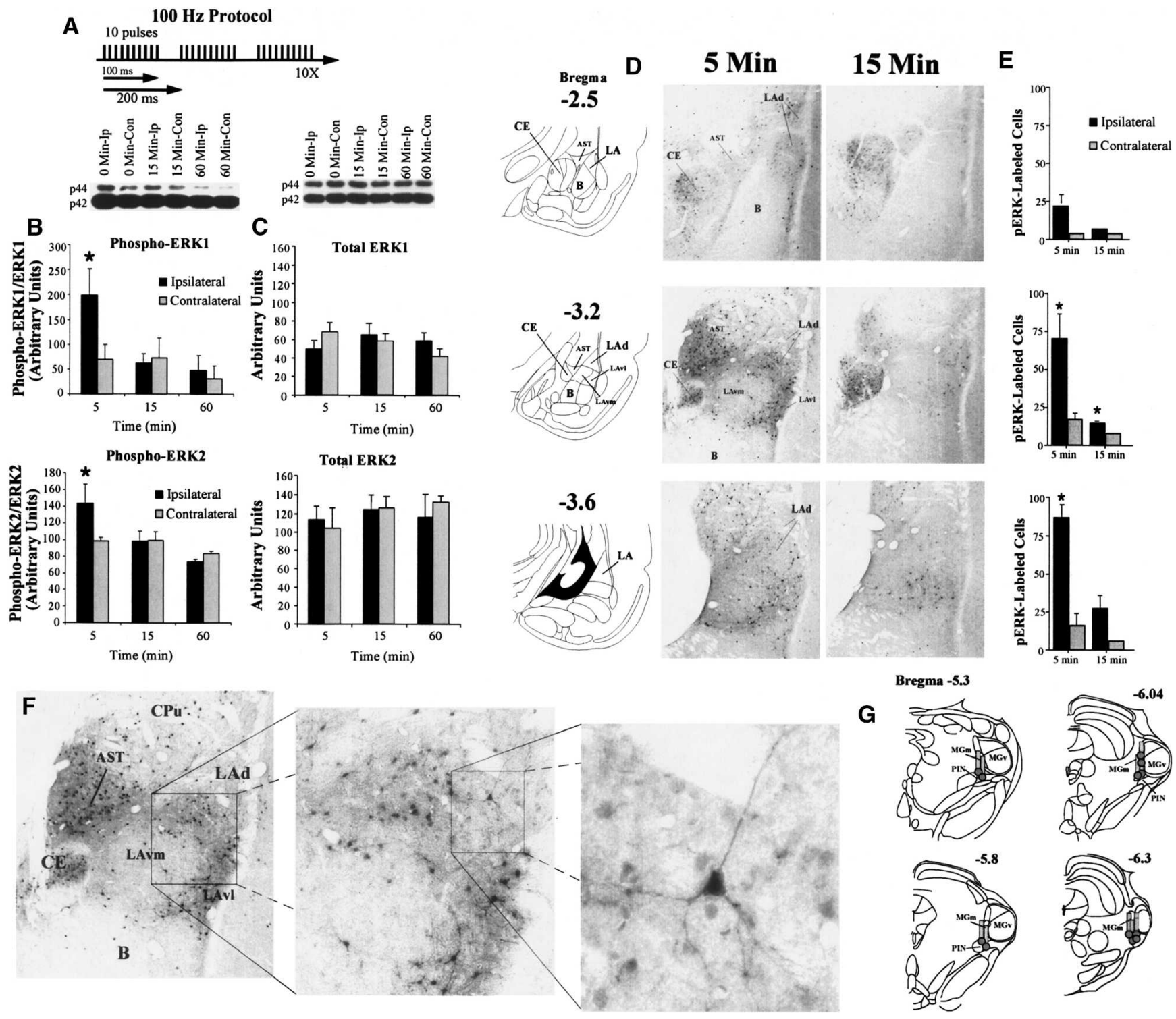

Figure 1. Regulation of ERK/MAPK in the LA after LTP-inducing stimulation, in vivo. (A) Anesthetized rats were given 100-Hz high-frequency stimulation (HFS) through an electrode implanted into MGm/PIN. (B) Significant activation of ERK1 (phospho-ERK1; top) and ERK2 (phospho-ERK2; bottom) was observed within 5 min after LTP- inducing stimulation but not at $t=15$ or 60 min after LTP. Data are shown for the amygdala ipsilateral (black bars) and contralateral (gray bars) to the stimulation ( $n=5$ /group). ${ }^{*} p<0.05$ relative to the contralateral side. In each experiment, levels of phospho-ERK were normalized to total ERK for each sample. Representative blots can be seen in the inset above. (C) Observed increase in ERK/MAPK activation following LTP-inducing stimulation is not due to a change in the total amount of either ERK1 (top) or ERK2 (bottom). Representative blots can be seen in the inset above. (D) Anatomical localization of cells expressing activated ERK/MAPK at 5 and 15 min after LTP-inducing stimulation at different anterior-posterior levels in the LA. Note the strong activation of ERK in the more ventral portions of the LAd, as well as the LAvl and amygdala-striatal transition zone (AST). This segregation of pERK label persists at all A-P levels. ( $E$ ) Cell counts of pERK-labeled cells taken at the three rostrocaudal levels represented in $D .{ }^{*} P<0.05$ relative to the contralateral side. $(F)$ Higher-level magnification images $(10 \times, 20 \times$, and $40 \times$, respectively) of activated ERK/MAPK in the LA after LTP-inducing stimulation. (G) Stimulation electrode placements in the MGm/PIN. 
and, to a lesser extent, ERK2 (phospho-ERK2; Fig. 1B, bottom) ipsilateral to the stimulation. Relative to the contralateral side of the brain, this activation was significantly different at $5 \mathrm{~min}$ after LTP-inducing stimulation (phospho-ERK1, $t_{(4)}=3.04, P<0.05$; phospho-ERK2, $\left.t_{(4)}=2.36, P<0.05\right)$ but failed to reach significance at $15 \mathrm{~min}$ (phospho-ERK1, $t_{(4)}=0.31, P>0.05$; phosphoERK2, $t(4)=0.05, P>0.05)$. At the 60 -min time point, no difference was observed for phospho-ERK1 $(t(4)=0.59, P>0.05)$. Phospho-ERK2 exhibited a very small yet significant reduction at 60 min relative to the contralateral side $(t(4)=3.58, P<0.05)$.

Importantly, pERK regulation following HFS was not accounted for by changes in total (unphosphorylated) ERK1 (Fig. 1C, top) or total ERK2 (Fig. 1C, bottom). No significant differences in total ERK were observed at $5 \mathrm{~min}$ (ERK1, $t_{(4)}=0.46$, $P>0.05$; ERK2, $\left.t_{(4)}=0.80, P>0.05\right), 15 \mathrm{~min}\left(\mathrm{ERK} 1, t_{(4)}=0.92\right.$, $P>0.05$; ERK2, $\left.t_{(4)}=0.93, P>0.05\right)$, or $60 \mathrm{~min}\left(\right.$ ERK1, $t_{(4)}=2.29$, $P>0.05$; ERK2, $\left.t_{(4)}=0.67, P>0.05\right)$ after LTP-inducing stimulation.

The findings of the immunohistochemical experiments mirrored those of the Western blotting (Fig. 1D-F) and also provided additional information about the anatomical localization of pERK following HFS. Relative to the contralateral side and to animals implanted only with the stimulation electrode (data not shown), HFS induced a robust activation of ERK in the LA that was most prominent at $5 \mathrm{~min}$ after the stimulation and present throughout the rostrocaudal extent of the LA (Fig. 1D,E). Analysis of cell counts (Fig. 1E) revealed a significant elevation in pERK-labeled cells ipsilateral to the stimulation in the group sacrificed at $5 \mathrm{~min}$, particularly in the middle $\left(\sim\right.$ bregma $-3.2 ; t_{(2)}=4.05$, $P<0.05$; middle panel) and posterior ( $\sim$ bregma -3.6$)$ portions of the LA $\left(t_{(2)}=30.05, P<0.05\right.$; bottom panel $)$. The anterior portion of the LA ( bregma -2.5 ; top panel) showed no significant regulation at this time point $\left(t_{(2)}=2.62\right.$, $P>0.05)$. At $15 \mathrm{~min}$, no significant differences were detected in the anterior $\left(t_{(2)}=2.03, P>0.05\right)$ or posterior $\left(t_{(2)}=2.14, P>0.05\right)$ regions of the LA. In the middle sections, however, a very small yet significant increase was observed $\left(t_{(2)}=5.58, P<0.05\right)$.

Interestingly, pERK label induced by HFS was largely restricted to the more ventral portions of the LAd and LAvl, regions that have previously been shown to exhibit regulation of pERK following fear conditioning (Schafe et al. 2000; Radwanska et al. 2002) and to exhibit long-term, extinction-resistant synaptic plasticity during fear learning (Repa et al. 2001). Significant pERK regulation was also observed in the amygdalastriatal transition zone (AST; Fig. 1D,F) and, to a lesser extent, in the LAvm, but not in the basal nucleus of the amygdala (B; Fig. 1D,F). The central nucleus of the amygdala (CE) was observed to exhibit high levels of pERK on both sides of the brain that did not appear to vary as a function of time (Fig. 1D).

Stimulation electrode placements can be viewed in Figure 1G. Electrode tips were located in most cases in medial portion of the PIN, with the tube extending into the MGm. Previous studies have shown that these regions of the auditory thalamus give rise to projections that terminate in the LA (Doron and LeDoux 1999).

\section{Phosphorylation of ERK/MAPK in the LA is specific to HFS}

In our previous experiment, we determined that HFS of thalamic inputs to the LA leads to increases in pERK ipsilateral to the stimulation. Here, we asked whether ERK regulation following thalamic stimulation was specific to HFS (e.g., LTP-inducing) stimulation. Rats received either HFS or low-frequency stimulation (LFS) of thalamic inputs to the LA as described below (Materials and Methods) and were sacrificed by decapitation or perfusion $\sim 5$ min thereafter.

Prior to running this experiment, we determined whether our LFS protocol (300 pulses over $2 \mathrm{~min}$ at $2.5 \mathrm{~Hz}$ ) did indeed produce little or no LTP in the LA (Fig. $2 \mathrm{~B}$ ). Relative to rats given
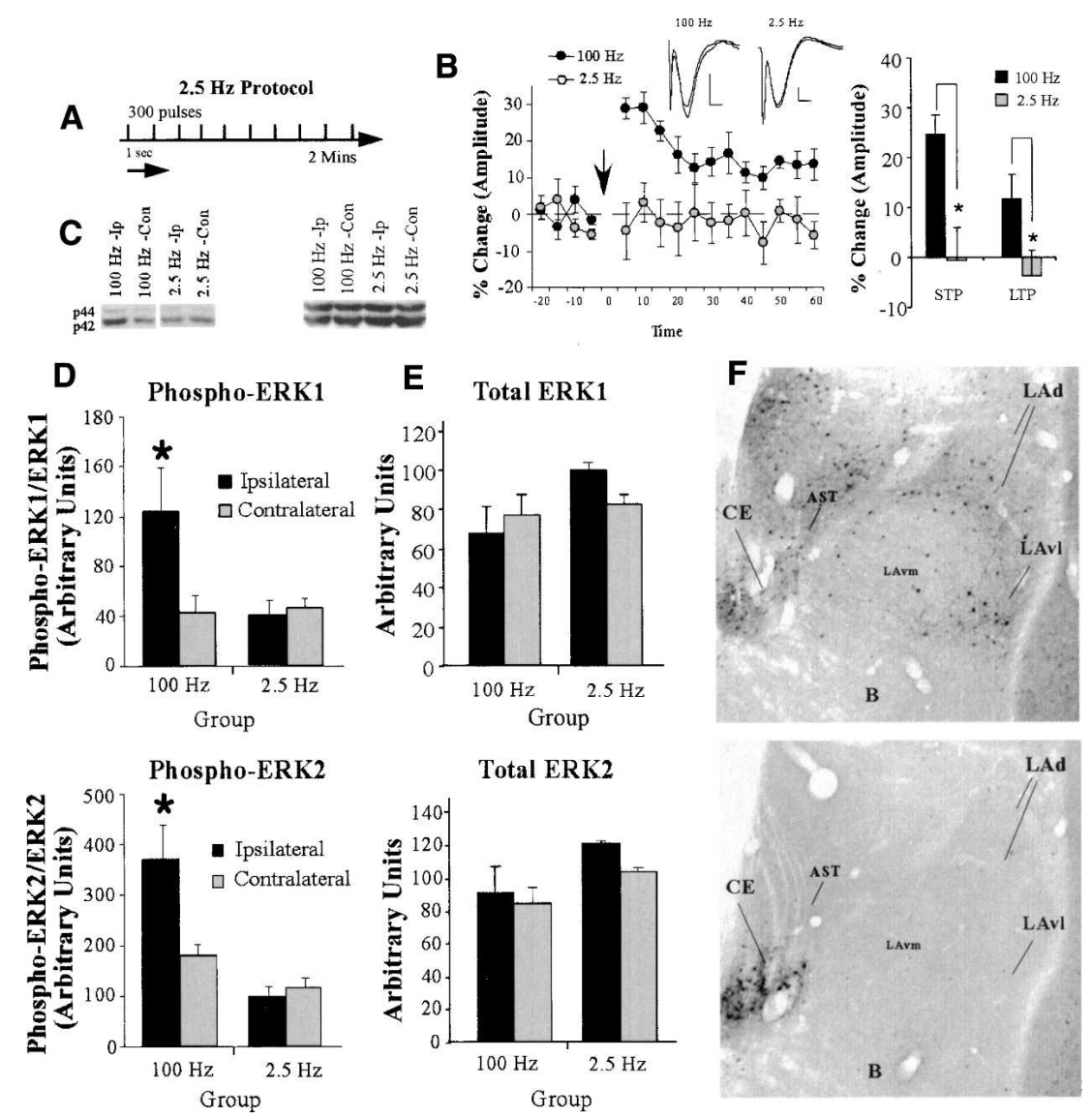

Figure 2. Regulation of ERK/MAPK in the LA after LTP-inducing stimulation is specific to HFS. ( $A$ ) Low-frequency stimulation (LFS) protocol. Rats received the same number of pulses (300) over the same time period (2 min) but at lower frequency $(2.5 \mathrm{~Hz})$. (B) Confirmation that HFS, but not LFS, induces significant LTP at thalamo-LA synapses. On the left, both groups (HFS, LFS) are represented across the entire 60 -min recording session (HFS, $n=5 ; \mathrm{LFS}, n=4)$. Representative traces for HFS and LFS can be seen in the inset (scale, $0.2 \mathrm{mV}$ by $5 \mathrm{msec}$ ). On the right, data are presented as percent change from baseline in the field potential amplitude during "STP," defined as the first 10 min after induction, and "LTP," defined as the last 10 min of the recording session. ${ }^{*} P<0.05$ relative to the HFS group. (C) Representative blots of phospho-ERK and total ERK in both HFS and LFS conditions. (D) HFS, but not LFS, of the MGm/PIN leads to significant elevations in activated ERK1 (top) and ERK2 (bottom) in the LA. ${ }^{*} P<0.05$ relative to the contralateral side ( $n=4 /$ group). ( $E$ ) No change in total ERK1 (top) or ERK2 (bottom) was observed. ( $F$ ) Representative photomicrographs from the LA after HFS (top) or LFS (bottom). Note the complete lack of ERK activation in the LA of the LFS animal. 
HFS, LFS failed to induce either short-term potentiation (STP, measured in the first $10 \mathrm{~min}$ following stimulation $\left(t_{(4)}=0.60\right.$, $P>0.05$, relative to baseline) or LTP (measured during the last 10 min of the recording session; $t_{(4)}=0.21, P>0.05$, relative to baseline). In contrast, the HFS protocol induced both STP $\left(t_{(5)}=3.97\right.$, $P<0.05)$ and $\operatorname{LTP}\left(t_{(5)}=7.36, P<0.05\right.$, both relative to baseline). Further, HFS and LFS groups differed significantly from one another both during STP $\left(t_{(9)}=4.58, P<0.05\right)$ and LTP $\left(t_{(9)}=3.34\right.$, $P<0.05)$.

The results of the Western blotting experiments are presented in Figure 2, D and E. As before, HFS induced a robust and significant activation of both ERK1 (phospho-ERK1, Fig. 2D, top; $t_{(3)}=3.68, P<0.05$ ) and ERK2 (phospho-ERK2, Fig. 2D, bottom; $t_{(3)}=2.36, P<0.05$ ), ipsilateral to the stimulation (relative to the contralateral side). In contrast, LFS did not lead to elevations in the phosphorylation of either ERK1 (Fig. 2D, top; $t_{(3)}=0.77$, $P>0.05$ ) or ERK2 (Fig. 2D, bottom; $t_{(3)}=0.18, P>0.05$ ). Also as before, no changes in total ERK1 or ERK2 were observed in rats receiving HFS (Fig. 1E, top and bottom; ERK1, $t_{(3)}=1.42$, $P>0.05$; ERK2, $\left.t_{(3)}=0.36, P>0.05\right)$.

Immunohistochemical localization of pERK following HFS and LFS can be seen in Figure 2F. As before, HFS induced a robust activation of ERK in the LA that was largely restricted to the more ventral portions of the LAd and LAvl (Fig. 2F, top). In contrast, LFS produced almost no pERK activation in the LA (Fig. 2F, bottom). As observed previously, the CE exhibited high levels of pERK that did not appear to vary as a function of stimulation condition (Fig. 2F).

Together with those of the first experiment, these findings indicate that LTP-inducing stimulation of the MGm/PIN induces a transient, anatomically restricted activation of ERK/MAPK in the LA.

\section{Intra-LA infusion of an ERK/MAPK inhibitor impairs LTP at thalamic inputs to the LA}

In our previous two experiments, we examined the regulation of ERK/MAPK in the LA following HFS of the MGm/PIN. Previous in vitro studies have shown that bath application of inhibitors of MEK, an upstream regulator of ERK/MAPK, impairs LTP at "thalamic" inputs to the LA (Huang et al. 2000; Schafe et al. 2000). Of particular interest is the finding that LTP in those experiments was impaired almost immediately following LTP induction under the influence of the MEK inhibitor, while in behavioral experiments the memory impairment induced by the same drug is evident only after many hours (that is, LTM is impaired at $\sim 24 \mathrm{~h}$ following training, but not STM, measured between 1 and $3 \mathrm{~h}$ after training; Schafe et al. 2000). Thus, despite clear correlation in mechanism between LTP and fear memory, these temporal discrepancies appear to present a challenge to the theory that LTP provides a neural substrate for LTM in the LA. One possibility is that this difference reflects something unique about the slice preparation. For example, neurons undergo significant trauma during preparation of brain slices for in vitro experiments, and they are disconnected from many of the modulatory inputs that are normally present in vivo. These factors may be responsible for quantitative differences in the time course of the effects of drugs on LTP and memory formation, even though both phenomena involve qualitatively similar molecular signaling pathways.

In this final experiment, we therefore asked whether activation of ERK in the LA following HFS is obligatory for LTP at thalamo-LA synapses, in vivo. To ask this question, we constructed a "cannula/electrode" consisting of an infusion cannula adhered to a steel recording wire which allowed for infusion of the MEK inhibitor U0126 $(1 \mu \mathrm{g} ; 0.5 \mu \mathrm{L})$ around the site of the recording (Fig. 3A; see Materials and Methods for details). This
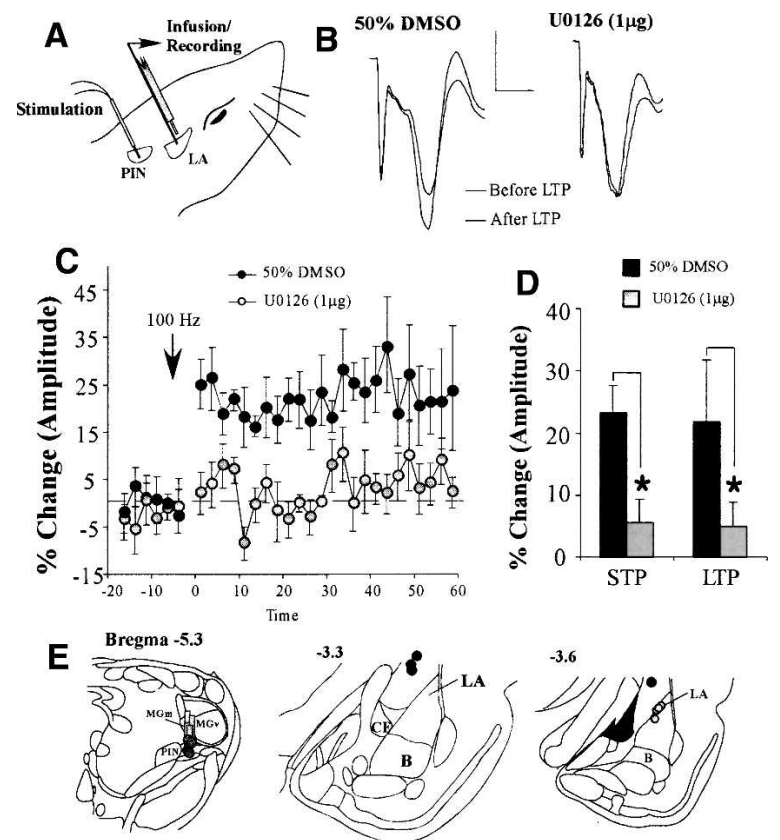

Figure 3. Impaired LTP at thalamic inputs, in vivo, by a localized infusion of an ERK/MAPK inhibitor in the LA. $(A)$ Anesthetized rats with a stimulation electrode implanted into the auditory thalamus and a recording cannula/electrode in the LA received HFS of the MGm/PIN 30 min after intra-amygdala infusion of either vehicle (50\% DMSO; $n=8)$ or the MEK inhibitor U0126 ( $1 \mu \mathrm{g} ; 0.5 \mu \mathrm{L} ; n=7)$. Recordings from the LA were made for $1 \mathrm{~h}$ following LTP induction. $(B)$ Representative field potentials in the LA evoked by MGm/PIN stimulation in vehicle and U0126 groups before and after LTP induction. Scale, $0.4 \mathrm{mV}$ by $5 \mathrm{msec}$. $(C, D)$ Infusion of U0126 (1 $\mu \mathrm{g}$; gray circles/bars) impaired LTP at thalamic inputs to the $\mathrm{LA}$, relative to vehicle-treated animals (black circles/bars). Here, "STP" is defined as the first 10 min after induction, while "LTP" is defined as the last $10 \mathrm{~min}$ of the recording session. ${ }^{*} P<0.05$ relative to vehicle. $(E)$ Cannula and recording electrode tip placements in or near the LA. Cannula tips are represented by the black circles; recording tips are represented by the gray circles.

dose of U0126 was chosen based on the findings of our previous work which showed that intra-LA infusion of U0126 produces a marked and dose-dependent reduction in ERK phosphorylation in LA homogenates following fear conditioning, with the 1- $\mu \mathrm{g}$ dose having the strongest effect (Schafe et al. 2000). Rats were given infusion of vehicle $(50 \%$ DMSO; $0.5 \mu \mathrm{L})$ or U0126 $30 \mathrm{~min}$ prior to HFS of the MGm/PIN. Recordings were made from the LA for $30 \mathrm{~min}$ following the infusion and for $1 \mathrm{~h}$ following LTP induction.

Results of the LTP experiment are presented Figure 3, C and D. Both STP and LTP at thalamic inputs to the LA were significantly impaired by U0126. Within the first $10 \mathrm{~min}$ of the recording session after LTP induction (STP; Fig. 3D), the vehicle group showed a $23 \pm 4 \%$ increase in field potential amplitude, which was significantly different from baseline $\left(t_{(7)}=6.38, P<0.05\right)$. In contrast, rats infused with U0126 showed $5.5 \pm 3 \%$ potentiation, which was not significantly different from baseline $\left(t_{(6)}=2.21, P>0.05\right)$. Within the last $10 \mathrm{~min}$ of the recording session (LTP; Fig. 3D), the vehicle group showed $22 \pm 10 \%$ increase in field potential amplitude, which was significantly different from baseline $\left(t_{(7)}=2.98, P<0.05\right)$. In contrast, rats infused with U0126 showed $5 \pm 4 \%$ potentiation, which was not significantly different from baseline $\left(t_{(6)}=2.16, P>0.05\right)$. Further, vehicle and U0126 groups differed significantly from one another during both the first $\left(t_{(13)}=3.29, P<0.05\right)$ and last 10 min of the recording session $\left(t_{(13)}=2.08, P<0.05\right)$. Thus, intra- 
LA infusion of U0126 at the dose that impairs fear memory consolidation (Schafe et al. 2000) also impairs LTP at thalamo-LA synapses in vivo.

During the 30-min period following infusions, we observed a reduction $(\sim 25 \%)$ in the amplitude of field-evoked responses in the LA (data not shown). Relative to the pre-infusion baseline, this reduction in amplitude was significant for both vehicle $\left(-26 \% ; t_{(7)}=3.19, P<0.05 ; n=8\right)$ and U0126-treated rats $\left(-22 \% ; t_{(6)}=5.64, P<0.05 ; n=7\right)$ in the 10 min prior to LTP induction. Importantly, however, there was no significant difference between vehicle and U0126-treated rats $\left(t_{(13)}=0.45\right.$, $P>0.05)$. Thus, while infusion of vehicle and drug solutions appeared to produce a small mechanical effect on the field-evoked potential in the LA, this effect is unlikely to account for the LTP impairment observed in U0126-treated rats.

Cannula and recording tip placements for the LTP experiment are presented in Figure 3E. The recording electrode tips were observed largely in the more ventral regions of the LAd, the same regions that exhibited significant regulation of ERK following HFS of the MGm/PIN in our stimulation experiments. Infusion cannula tips were observed in the ventral striatum, $\sim 0.5 \mathrm{~mm}$ above the recording sites.

\section{Discussion}

Previous studies that have examined the biochemical mechanisms of LTP in the amygdala have largely done so using pharmacological methods and in vitro slice recordings. Here, we have shown that LTP-inducing stimulation of the MGm/PIN induces ERK/MAPK regulation in anatomically defined regions of the LA and that ERK activation in the LA is required for LTP at thalamoLA synapses, in vivo.

Our experiments have a number of advantages over previous in vitro studies that have shown impairments of amygdala LTP under the influence of inhibitors of ERK/MAPK (Huang et al. 2000; Schafe et al. 2000). Principal among these is the ability to unambiguously examine the biochemical mechanisms and underlying pharmacology of LTP at genuine thalamo-LA synapses. While the amygdala slice preparation is convenient for pharmacological and whole-cell recording methods, the source of the inputs stimulated during a slice LTP experiment in the amygdala is not well defined. For example, a typical amygdala-slice experiment employs stimulation of either "cortical" or "thalamic" input pathways to the LA not by stimulating the thalamus or cortex proper but instead by stimulating fibers coursing through the external or internal capsules, respectively, that are known from anatomical studies to originate in these two structures (Chapman et al. 1990; Huang and Kandel 1998; Weisskopf and LeDoux 1999). Anatomical evidence, for example, supports the notion that auditory thalamic afferents travel rostrally toward the LA in the cerebral peduncle. At the level of the posterior amygdala, these fibers can be seen as a bundle running parallel to the medial extent of the optic tract before turning laterally and coursing through the ventral striatum prior to terminating in the LAd (LeDoux et al. 1990). As such, stimulation of these fibers just medial to the LA is believed to mimic stimulation of MGm/PIN itself, but likely also stimulates fibers originating in other thalamic nuclei and subcortical structures. A similar argument can be made for stimulation of the external capsule during "cortical LTP," which, in the coronal slice, likely involves stimulation of fibers originating not only in auditory cortex but from multiple cortical areas in the forebrain. While the horizontal slice procedure has been shown to more accurately preserve most of the connections between entorhinal and perirhinal cortex and LA (von Bohlen und Halbach and Albrecht 2002), allowing for direct cortico-LA stimulation, we know of no comparable preparation for the thalamo-LA pathway. As such, our in vivo study represents the first unambiguous demonstration that LTP at thalamic auditory inputs to the LA is dependent on ERK/MAPK activation.

A second major advantage of the in vivo LTP approach is that we have been able to examine the anatomical localization of pERK along the entire rostrocaudal extent of the LA after LTPinducing stimulation in a manner that would not have been possible using in vitro methods. In our experiments, ERK activation was observed in anatomically restricted regions of the LA, most notably in the more ventral portions of the LAd and LAvl and less so in the LAvm. These more ventral regions of the LAd and LAvl have previously been shown to be the recipients of the majority of terminal labeling from cells originating in the MGm/ PIN (Farb and LeDoux 1997). Importantly, this localization of pERK in the LA, while naturally more intense due to the artificial nature of the HFS, is in striking parallel to that which has previously been observed following auditory fear conditioning (Schafe et al. 2000). It is also in agreement with a recent neurophysiological study showing that cells in the more ventral regions of the LA exhibit synaptic plasticity during and after fear conditioning that is long-lasting and extinction resistant (Repa et al. 2001), a finding which suggests that these more ventrally located cells may be critical mediators of ERK-dependent long-term fear memory formation.

While it appears that LTP at thalamic inputs to the LA and fear conditioning both require ERK activation in the same anatomically restricted regions of the LA, there are also a number of interesting differences between amygdala LTP and fear conditioning. It is clear from previous in vitro studies, for example, that the decay of LTP under the influence of MEK inhibitors is faster than the decay of memory under the influence of the same drug (Huang et al. 2000; Schafe et al. 2000). In slice experiments, for example, an impairment of LTP at thalamic inputs to the LA has been observed, to varying degrees, in both STP and LTP (Huang et al. 2000; Schafe et al. 2000), while auditory fear memories under the influence of U0126 are intact at 1 and $3 \mathrm{~h}$ following training and only begin to show impairment beginning at 6 $\mathrm{h}$ (Schafe et al. 2000). This same pattern of findings also observed in mice lacking Ras-GRF, an upstream regulator of ERK/MAPK signaling (Brambilla et al. 1997). In that study, LTP in slices taken from Ras-GRF knockout mice is completely decayed back to baseline by $30 \mathrm{~min}$, while memory is intact out to at least $1 \mathrm{~h}$ (Brambilla et al. 1997). In our in vivo experiments, we observed a similarly rapid impairment of LTP following intra-LA infusion of U0126 (Fig. 3C). Further, in our biochemical experiments, ERK activation peaked within 5 min after HFS and was not observed to be regulated at either 15 or 60 min after LTP-inducing stimulation (Fig. 1B). This pattern of findings also stands in contrast to studies of fear conditioning, where ERK activation has been observed to exhibit a biphasic regulation both immediately after training and also 60 min later (Schafe et al. 2000; Paul et al. 2007). Clearly, these findings suggest that, while amygdala LTP and fear conditioning share a common biochemical substrateand perhaps engage plasticity in a common population of cells in the LA-they are not synonymous processes. It remains unclear at present what might account for such discrepancies between LTP and memory formation. Given that urethane anesthesia has been shown to impair the activation of ERK under some experimental conditions (Cancedda et al. 2003), it remains possible that we failed to observe a second peak of ERK phosphorylation due to the use of the anesthetized preparation. Alternatively, LTP-induced ERK activation may indeed peak solely within the first $5 \mathrm{~min}$ after induction, and the dual peaks of ERK phosphorylation after training may reflect multiple events of LTP-like synaptic plasticity occurring in the LA following learning, with each event contributing to the long-term consolidation process. The 
immediate impairment of LTP by MEK inhibitors, however, is less easy to explain and may represent an artifact of the artificial nature of LTP-inducing stimulation which recruits a cellular process that is not present during natural learning. Importantly, recent studies that have examined training-induced neural plasticity and memory formation in the LA and their joint reliance on ERK/MAPK have shown that the two processes occur and decay in parallel (Schafe et al. 2005b). Accordingly, the awake in vivo recording technique may hold greater promise in linking synaptic plasticity and memory formation.

In summary, our findings show that ERK/MAPK activation is required for LTP in anatomically defined regions of the LA, in vivo. While a number of interesting differences remain between LTP and learning, our study demonstrates the utility of the in vivo LTP preparation for driving synaptic plasticity and intracellular signaling pathways at large numbers synapses in regions of the LA that also exhibit significant biochemical changes following fear conditioning. This method has clear advantages for further examining the molecular basis of fear memory formation in the amygdala.

\section{Materials and Methods}

\section{Subjects}

Adult male, experimentally naive Sprague-Dawley rats (Hilltop Lab Animals, Inc.) were housed individually in plastic Nalgene cages and maintained on a $12 \mathrm{~h}: 12 \mathrm{~h}$ light/dark cycle. Food and water were provided ad libitum throughout the experiment. All procedures were in accordance with the National Institutes of Health Guide for the Care and Use of Experimental Animals and were approved by the New York University Animal Care and Use Committee.

\section{Electrophysiology}

Rats (300-350 g) were anesthetized with urethane (two i.p. injections at $10-\mathrm{min}$ intervals; total of $1.6 \mathrm{mg} / \mathrm{kg}$ ) and placed in a stereotaxic frame. The skull was exposed over the auditory thalamus (medial geniculate nucleus and posterior intralaminar nucleus; MGm/PIN), the dura was retracted, and rats were implanted with a bipolar stimulating electrode into the MGm/PIN. Coordinates for MGm/PIN were 5.0 AP, 2.9 ML, -6.6 DV. Onehalf hour following implantation of the stimulating electrode, rats were given LTP-inducing (high-frequency) stimulation (HFS) consisting of three series of theta-patterned $100-\mathrm{Hz}$ tetani given once a minute at a stimulation intensity of $300 \mu \mathrm{A}, 100 \mu \mathrm{sec}$ (Fig. 1A). This intensity, which is within the range of our own LTP experiments (see below) and similar, if not lower, than that previously used in recording studies in anesthetized (Clugnet and LeDoux 1990; Rogan and LeDoux 1995; Yaniv et al. 2001) and awake-behaving animals (Doyère et al. 2003), was chosen as it is likely to induce LTP in every animal without producing seizures. In some experiments, additional controls were included that received implantation of the electrode only without additional stimulation, or with low-frequency stimulation (LFS). The LFS group received the same total number of pulses as rats in the LTP group (300 total pulses over $2 \mathrm{~min}$ ) but at lower frequency ( 2.5 Hz; Fig. 2A).

For standard LTP recording experiments (Fig. 2B), rats were anesthetized as before and implanted with a stimulation electrode in the MGm/PIN and a glass recording pipette $(0.5-8 \mathrm{M} \Omega$ ) into the LA (AP $-3.2, \mathrm{ML}+5.5, \mathrm{DV}-6.0$ ) as previously described (Apergis-Schoute et al. 2005). The recording pipette was filled with $150 \mathrm{mM}$ sodium acetate/2.5\% pontamine sky blue ( $\mathrm{pH} \mathrm{8.0)}$. Stimulation of the MGm/PIN was observed to evoke field potentials in the LA, with a characteristic negative-going component of $\sim 5-10$ msec which corresponds to the peak of multi-unit activity (Doyère et al. 2003). Field-evoked potentials in the LA were recorded at $0.03 \mathrm{~Hz}$ for $1 \mathrm{~h}$ to establish a stable baseline. In these experiments, it was not desirable to keep the simulation intensity constant at $300 \mu \mathrm{A}$ as we did in our stimulation experiments, since the measurement of LTP requires that a field-evoked response fall within the dynamic range of intensity, a value which varies for each experiment. As such, for each experiment a test intensity was chosen that evoked a field potential that was approximately half of its maximal amplitude $(100-400 \mu \mathrm{A} ; 100$ usec). Following baseline recording, rats received either HFS or LFS of the MGm/PIN at baseline intensity, and recordings were resumed for an additional hour. Field-evoked responses in the LA were digitized using a CED Micro 1401 interface and Spike2 software (Cambridge Electronic Design). The amplitude of individual field potentials was measured using Spike2 and normalized to the mean amplitude during the 10-min period immediately preceding HFS or LFS. Data were analyzed during the first (STP) and last (LTP) 10 min of the recording session and analyzed using Student's $t$-tests (HFS vs. LFS) or paired $t$-tests (either group relative to baseline).

For LTP infusion experiments (Fig. 3), rats were implanted with a stimulation electrode into the MGm/PIN and a steel recording electrode into the LA that was adhered to a 26-gauge stainless steel infusion cannula (Fig. 3A). This "cannula/ electrode" preparation was fitted with a 33-gauge infusion cannula that was attached to a syringe pump for drug delivery into the brain. The electrode tip extended $\sim 0.5 \mathrm{~mm}$ below the tip of the infusion cannula to minimize mechanical effects of the infusion on the recording. In these experiments, rats were given 1 $\mathrm{h}$ of baseline recording $(0.03 \mathrm{~Hz})$ followed by infusion of either $50 \%$ DMSO $(0.5 \mu \mathrm{L})$ or $1 \mu \mathrm{g}$ of U0126 (Promega) in a $0.5-\mu \mathrm{L}$ volume of $50 \%$ DMSO. The rate of infusion was $\sim 0.05 \mu \mathrm{L} / \mathrm{min}$. This concentration and volume of DMSO are required to solubilize U0126 in aqueous solution, although other laboratories have also found a $20 \%$ DMSO solution to be effective (Steward et al. 2007). Importantly, the $50 \%$ DMSO vehicle has been used successfully in a number of experiments for local infusion into the amygdala without apparent toxicity or functional effects on neurophysiological function (Schafe et al. 2000, 2005a,b; Duvarci et al. 2005; Doyère et al. 2007). At the end of the infusion period, rats were given a second baseline recording for $30 \mathrm{~min}$, followed by HFS of MGm/PIN (see protocol above). Following the tetanus, recordings were resumed for $1 \mathrm{~h}$. Data were analyzed during the first (STP) and last (LTP) $10 \mathrm{~min}$ of the recording session and analyzed using Student's $t$-tests (drug vs. vehicle) or paired $t$-tests (either group relative to baseline).

\section{Western blotting}

For Western blotting experiments, rats received HFS or LFS of thalamic inputs to the LA as described above and were sacrificed by decapitation at the appropriate time interval after stimulation. Brains were rapidly removed from the skull, frozen, and stored at $-80^{\circ} \mathrm{C}$ until processed. Amygdala punches were obtained with a 1 -mm punch tool (Fine Science Tools) from $400-\mu \mathrm{m}$-thick frozen sections taken on a sliding freezing microtome. The punches included the LA and the basal nucleus. Punches were briefly manually homogenized in $200 \mu \mathrm{L}$ of ice-cold lysis buffer containing $20 \mathrm{mM}$ Tris- $\mathrm{HCl}$ (pH 7.5), $1 \mathrm{mM}$ EGTA, $1 \mathrm{mM}$ EDTA, $25 \mu \mathrm{g} / \mathrm{mL}$ aprotinin, $25 \mathrm{\mu g} / \mathrm{mL}$ leupeptin, $1 \mathrm{mM}$ sodium pyrophosphate, $500 \mu \mathrm{M}$ phenylmethylsulfonyl fluoride, $4 \mathrm{mM}$ para-nitrophenyl phosphate, and $1 \mathrm{mM}$ sodium orthovanadate. Prior to adding sample buffer, a Bradford assay was run to determine the amount of protein in each sample, and samples were adjusted with lysis buffer so that each sample contained the same amount of protein. Immediately following the protein assay, sample buffer was added to the homogenates, and the samples were boiled for 3-4 min. Homogenates were electrophoresed on $7.5 \%$ SDSpolyacrylamide gels and blotted to PVDF paper (Millipore). Western blots were blocked in TTBS buffer $(50 \mathrm{mM}$ Tris- $\mathrm{HCl}$ at $\mathrm{pH} 7.5$, $150 \mathrm{mM} \mathrm{NaCl}, 0.05 \%$ Tween 20) with $3 \%$ milk then incubated with an anti-phospho-ERK/MAPK (pERK; 1:1000; New England Biolabs). Blots were then incubated with anti-rabbit conjugated to horseradish peroxidase (UBI) and developed using enhanced chemiluminescence (Amersham). Western blots were developed 
in the linear range by taking multiple exposures of both ERK1 and ERK2, and densitometry was conducted using either ImagePro software (Fig. 1) or ImageJ software (Fig. 2). In all experiments, ipsilateral samples were always loaded adjacent to contralateral samples from the same animal, and each condition (time, HFS vs. LFS) was represented on the same blot. For analysis, we compared regulation of pERK ipsilateral to the stimulation relative to the contralateral (nonstimulated) side using paired $t$-tests. Following analysis of pERK, blots were stripped with $0.2 \mathrm{M}$ $\mathrm{NaOH}$ and reprobed with an antibody directed against total (unphosphorylated) ERK/MAPK (1:1000; Upstate Biotechnology, Inc.) and processed and quantified as before. In all experiments, pERK levels were expressed relative to total ERK levels.

\section{Immunohistochemistry}

For immunohistochemical experiments, rats received HFS or LFS of the MGm/PIN as described above and, at the appropriate time interval, were perfused with ice-cold phosphate-buffered saline (PBS) followed by ice-cold $4 \%$ paraformaldehyde in $0.1 \mathrm{M}$ phosphate buffer (PB). Brains were removed and post-fixed in $4 \%$ paraformaldehyde/PB for $24 \mathrm{~h}$ and then cryoprotected in $20 \%$ glycerol/0.1 M PB for $48 \mathrm{~h}$ Immunohistochemistry was carried out as previously described (Schafe et al. 2000; Swank 2000). Briefly, $40-\mu \mathrm{m}$ free-floating sections through the amygdala were obtained using a sliding microtome. Every third section was processed for pMAPK immunoreactivity. After blocking in Trisbuffered saline (TBS) containing $4 \%$ normal goat serum $/ 0.2 \%$ Triton X-100, slices were incubated at $4^{\circ} \mathrm{C}$ for $72 \mathrm{~h}$ in antiphospho-MAPK (rabbit polyclonal antibody, 1:4000, New England Biolabs) in TBS/2\% goat serum/0.1\% Triton. Following extensive washes in TBS, tissue sections were incubated in biotinylated goat anti-rabbit (pre-diluted, Kirkegaard \& Perry Labs/KPL) for $2 \mathrm{~h}$ at room temperature. Tissue was again rinsed in TBS followed by incubation in streptavidin-HRP (KPL) at room temperature for $1 \mathrm{~h}$, rinsed in TBS, and developed in cobalt-enhanced DAB (Enhance Black, KPL) for $10 \mathrm{~min}$. Sections were mounted on subbed slides and coverslipped.

\section{Quantification of pMAPK-labeled cells}

Sections from three different anterior-posterior levels were selected for scoring, $\sim 2.5,3.2$, or $3.6 \mathrm{~mm}$ posterior to bregma. Cell counts were taken from at least three sections per rat and scored using a defined boundary roughly equivalent to the size of the LA using ImageJ software. For analysis, we compared regulation of pERK ipsilateral to the stimulation relative to the contralateral (nonstimulated) side using paired $t$-tests. Since every third section through the amygdala was processed for immunohistochemistry, it was not necessary to correct for double-counting.

\section{Acknowledgments}

We thank Yu Zhou for assistance in making the electrodes. This research was supported in part by National Institute of Mental Health grants (MH 62519, MH 46516, MH 00956, MH 39774, and MH 11902) and by the Centre National de la Recherche Scientifique (CNRS)-NSF (grant 17089), CNRS-PICS, and EU-FP6 PROMEMORIA.

\section{References}

Apergis-Schoute, A.M., Debiec, J., Doyere, V., LeDoux, J.E., and Schafe, G.E. 2005. Auditory fear conditioning and long-term potentiation in the lateral amygdala require ERK/MAP kinase signaling in the auditory thalamus: A role for presynaptic plasticity in the fear system. J. Neurosci. 25: 5730-5739.

Bauer, E.P., LeDoux, J.E., and Nader, K. 2001. Fear conditioning and LTP in the lateral amygdala are sensitive to the same stimulus contingencies. Nat. Neurosci. 4: 687-688.

Bauer, E.P., Schafe, G.E., and LeDoux, J.E. 2002. NMDA receptors and L-type voltage-gated calcium channels contribute to long-term potentiation and different components of fear memory formation in the lateral amygdala. J. Neurosci. 22: 5239-5249.
Blair, H.T., Schafe, G.E., Bauer, E.P., Rodrigues, S.M., and LeDoux, J.E. 2001. Synaptic plasticity in the lateral amygdala: A cellular hypothesis of fear conditioning. Learn. Mem. 8: 229-242.

Brambilla, R., Gnesutta, N., Minichiello, L., White, G., Roylance, A.J., Herron, C.E., Ramsey, M., Wolfer, D.P., Cestari, V., Rossi-Arnaud, C., et al. 1997. A role for the Ras signalling pathway in synaptic transmission and long-term memory. Nature 390: 281-286.

Cancedda, L., Putignano, E., Impey, S., Maffei, L., Ratto, G.M., and Pizzorusso, T. 2003. Patterned vision causes CRE-mediated gene expression in the visual cortex through PKA and ERK. J. Neurosci. 23: 7012-7020.

Chapman, P.F., Kairiss, E.W., Keenan, C.L., and Brown, T.H. 1990. Long-term synaptic potentiation in the amygdala. Synapse 6: $271-278$.

Clugnet, M.C. and LeDoux, J.E. 1990. Synaptic plasticity in fear conditioning circuits: induction of LTP in the lateral nucleus of the amygdala by stimulation of the medial geniculate body. J. Neurosci. 10: $2818-2824$

Doron, N.N. and LeDoux, J.E. 1999. Organization of projections to the lateral amygdala from auditory and visual areas of the thalamus in the rat. J. Comp. Neurol. 412: 383-409.

Doyère, V., Schafe, G.E., Sigurdsson, T., and LeDoux, J.E. 2003. Long-term potentiation in freely moving rats reveals asymmetries in thalamic and cortical inputs to the lateral amygdala. Eur. J. Neurosci. 17: 2703-2715.

Doyère, V., Dȩbiec, J., Monfils, M.H., Schafe, G.E., and LeDoux, J.E. 2007. Synapse-specific reconsolidation of distinct fear memories in the lateral amygdala. Nat. Neurosci. 10: 414-416.

Duvarci, S., Nader, K., and LeDoux, J.E. 2005. Activation of extracellular signal-regulated kinase/mitogen-activated protein kinase cascade in the amygdala is required for memory reconsolidation of auditory fear conditioning. Eur. J. Neurosci. 21: 283-289.

Farb, C.R. and LeDoux, J.E. 1997. NMDA and AMPA receptors in the lateral nucleus of the amygdala are postsynaptic to auditory thalamic afferents. Synapse 27: 106-121.

Huang, Y.Y. and Kandel, E.R. 1998. Postsynaptic induction and PKA-dependent expression of LTP in the lateral amygdala. Neuron 21: $169-178$.

Huang, Y.Y., Martin, K.C., and Kandel, E.R. 2000. Both protein kinase A and mitogen-activated protein kinase are required in the amygdala for the macromolecular synthesis-dependent late phase of long-term potentiation. J. Neurosci. 20: 6317-6325.

LeDoux, J.E., Farb, C., and Ruggiero, D.A. 1990. Topographic organization of neurons in the acoustic thalamus that project to the amygdala. J. Neurosci. 10: 1043-1054.

Maren, S. 1999. Long-term potentiation in the amygdala: A mechanism for emotional learning and memory. Trends Neurosci. 22: 561-567.

Maren, S. and Fanselow, M.S. 1995. Synaptic plasticity in the basolateral amygdala induced by hippocampal formation stimulation in vivo. $J$. Neurosci. 15: 7548-7564.

McKernan, M.G. and Shinnick-Gallagher, P. 1997. Fear conditioning induces a lasting potentiation of synaptic currents in vitro. Nature 390: 607-611.

Paul, S., Olausson, P., Venkitaramani, D.V., Ruchkina, I., Moran, T.D., Tronson, N., Mills, E., Hakim, S., Salter, M.W., Taylor, J.R., et al. 2007. The striatal-enriched protein tyrosine phosphatase gates long-term potentiation and fear memory in the lateral amygdala. Biol. Psychiatry 61: 1049-1061.

Radwanska, K., Nikolaev, E., Knapska, E., and Kaczmarek, L. 2002. Differential response of two subdivisions of lateral amygdala to aversive conditioning as revealed by c-Fos and P-ERK mapping. Neuroreport 13: 2241-2246.

Repa, J.C., Muller, J., Apergis, J., Desrochers, T.M., Zhou, Y., and LeDoux, J.E. 2001. Two different lateral amygdala cell populations contribute to the initiation and storage of memory. Nat. Neurosci. 4: 724-731.

Rogan, M.T. and LeDoux, J.E. 1995. LTP is accompanied by commensurate enhancement of auditory-evoked responses in a fear conditioning circuit. Neuron 15: 127-136.

Rogan, M.T., Staubli, U.V., and LeDoux, J.E. 1997. Fear conditioning induces associative long-term potentiation in the amygdala. Nature 390: 604-607.

Schafe, G.E., Atkins, C.M., Swank, M.W., Bauer, E.P., Sweatt, J.D., and LeDoux, J.E. 2000. Activation of ERK/MAP kinase in the amygdala is required for memory consolidation of Pavlovian fear conditioning. $J$. Neurosci. 20: 8177-8187.

Schafe, G.E., Nader, K., Blair, H.T., and LeDoux, J.E. 2001. Memory consolidation of Pavlovian fear conditioning: A cellular and molecular perspective. Trends Neurosci. 24: 540-546.

Schafe, G.E., Bauer, E.P., Rosis, S., Farb, C.R., Rodrigues, S.M., and LeDoux, J.E. 2005a. Memory consolidation of Pavlovian fear conditioning requires nitric oxide signaling in the lateral amygdala. 
Eur. J. Neurosci. 22: 201-211.

Schafe, G.E., Doyere, V., and LeDoux, J.E. 2005b. Tracking the fear engram: The lateral amygdala is an essential locus of fear memory storage. J. Neurosci. 25: 10010-10014.

Steward, O., Huang, F., and Guzowski, J.F. 2007. A form of perforant path LTP can occur without ERK1/2 phosphorylation or immediate early gene induction. Learn. Mem. 14: 433-445.

Swank, M.W. 2000. Phosphorylation of MAP kinase and CREB in mouse cortex and amygdala during taste aversion learning. Neuroreport 11: $1625-1630$.

von Bohlen und Halbach, O. and Albrecht, D. 2002. Reciprocal connections of the hippocampal area CA1, the lateral nucleus of the amygdala and cortical areas in a combined horizontal slice preparation. Neurosci. Res. 44: $91-100$.

Weisskopf, M.G. and LeDoux, J.E. 1999. Distinct populations of NMDA receptors at subcortical and cortical inputs to principal cells of the lateral amygdala. J. Neurophysiol. 81: 930-934.

Yaniv, D., Schafe, G.E., LeDoux, J.E., and Richter-Levin, G. 2001. A gradient of plasticity in the amygdala revealed by cortical and subcortical stimulation, in vivo. Neuroscience 106: 613-620.

Received August 20, 2007; accepted in revised form November 25, 2007. 


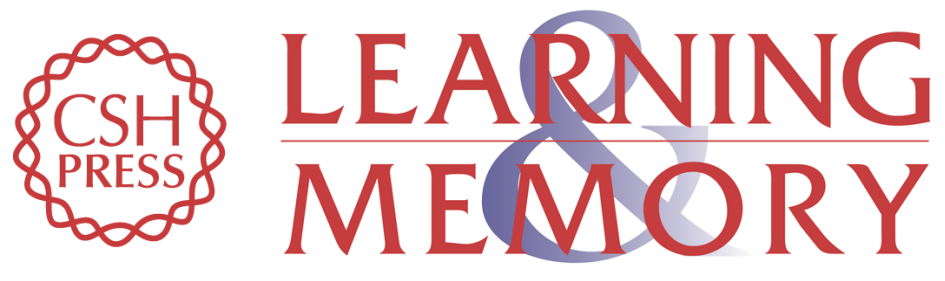

\section{Phosphorylation of ERK/MAP kinase is required for long-term potentiation in anatomically restricted regions of the lateral amygdala in vivo}

Glenn E. Schafe, Michael W. Swank, Sarina M. Rodrigues, et al.

Learn. Mem. 2008, 15:

Access the most recent version at doi:10.1101//m.746808

References This article cites 33 articles, 11 of which can be accessed free at:

http://learnmem.cshlp.org/content/15/2/55.full.html\#ref-list-1

License

Email Alerting

Receive free email alerts when new articles cite this article - sign up in the box at the Service top right corner of the article or click here. 